\title{
Measurements of plasma spectra from hot dense elements and mixtures at conditions relevant to the solar radiative zone
}

D. J. Hoarty, E. Hill, P. Beiersdorfer, P. Allan, C. R. D. Brown, M. P. Hill, L. M. R. Hobbs, S. F. James, J. Morton,

N. Sircombe, L. Upcraft, J. W. O. Harris, R. Shepherd, E. Marley, E. Magee, J. Emig, J. Nilsen, and S. J. Rose

Citation: AIP Conference Proceedings 1811, 050001 (2017); doi: 10.1063/1.4975721

View online: http://dx.doi.org/10.1063/1.4975721

View Table of Contents: http://aip.scitation.org/toc/apc/1811/1

Published by the American Institute of Physics

\section{Articles you may be interested in}

Calculation of atomic structures and radiative properties of fusion plasmas

AIP Conference Proceedings 1811, 070002070002 (2017); 10.1063/1.4975728

Stark broadening of hydrogen lines in magnetic fusion plasmas

AIP Conference Proceedings 1811, 100001100001 (2017); 10.1063/1.4975732

Turbulence and atomic physics in magnetically confined plasmas

AIP Conference Proceedings 1811, 090001090001 (2017); 10.1063/1.4975731

Non-LTE modeling of radiatively driven dense plasmas

AIP Conference Proceedings 1811, 050004050004 (2017); 10.1063/1.4975724 


\title{
Measurements of plasma spectra from hot dense elements and mixtures at conditions relevant to the solar radiative zone.
}

\author{
D J Hoarty, a), E Hill ${ }^{2}$, P Beiersdorfer ${ }^{3}$, P Allan ${ }^{1}$, C R D Brown ${ }^{1}$, M P Hill ${ }^{1}$, L M R \\ Hobbs $^{1}$, S F James ${ }^{1}$, J Morton ${ }^{1}$, N Sircombe ${ }^{1}$, L Upcraft ${ }^{1}$, J W O Harris ${ }^{1}$, R

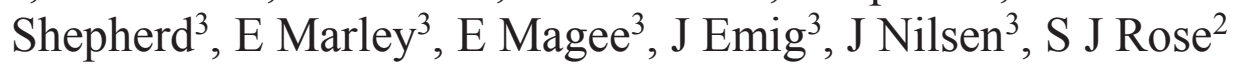 \\ ${ }^{1}$ Materials and Computational Physics, AWE plc, Reading, RG74PR, UK. \\ ${ }_{2}^{2}$ Plasma Physics Group, Blackett Laboratory, Imperial College London, London, SW7 2AZ, UK. \\ ${ }^{3}$ Lawrence Livermore National Laboratory, Livermore, CA 94550, USA. \\ a) Corresponding author: David.Hoarty@awe.co.uk
}

\begin{abstract}
X-ray emission spectroscopy has been used to study hot dense plasmas produced using high power laser irradiation of dot samples buried in low $\mathrm{Z}$ foils of plastic or diamond. By combining a high contrast short pulse (picosecond timescale) laser beam operating in second harmonic with long pulse (nanosecond timescale) laser beams in third harmonic, and with pulse shaping of the long pulse beams, a range of plasma temperatures from $400 \mathrm{eV}$ up to $2.5 \mathrm{keV}$ and electron densities from $5 \mathrm{e} 22$ up to $1 \mathrm{e} 24 / \mathrm{cc}$ have been accessed. Examples are given of measurements of dense plasma effects such as ionization potential depression and line-broadening from the K-shell emission spectra of a range of low $\mathrm{Z}$ elements and mixtures and compared to model prediction. Detailed spectra from measurements of the Lshell emission from mid-Z elements are also presented for an example spectrum of germanium. These data are at conditions found in stellar interiors and in particular in the radiative zone of the sun. The plasma conditions are inferred from comparison of the measured spectra to detailed modeling using atomic kinetics and spectral synthesis codes.
\end{abstract}

\section{INTRODUCTION}

Dense plasma spectroscopy is widely used to diagnose plasma conditions in studies of properties such as opacity and equation of state [1]; in energy transport and in inertial fusion experiments [2]. These quantities are fundamental to the modelling of stellar interiors [3] and in the design of inertial confinement fusion schemes [4]. With the advent of high power short pulse lasers measurements to test the validity of models of dense plasma properties and spectral synthesis have been possible near solid density [5-10]. On the Orion laser system at AWE in the UK a combination of very high contrast short pulse, $0.7 \mathrm{ps}$, laser heating and compression using long pulse , $0.5 \mathrm{~ns}$, beams has allowed the density in these studies to be increased to significantly greater than solid density and the temperature to greater than that found at the solar core. Of particular interest are conditions in the radiative zone of the sun where energy is transferred from the core outwards by radiative diffusion. Conditions in the radiative zone range from $20-30 \mathrm{~g} / \mathrm{cc}, 700-800 \mathrm{eV}$ at the core boundary to around $200 \mathrm{eV}$ and $0.2 \mathrm{~g} / \mathrm{cc}$ at the convective zone boundary. The opacity of iron and argon are important in the radiation flow through this region and in part of this work elements similar in atomic number are studied, germanium and chlorine respectively, which exhibit similar spectra at the experimental conditions to those of iron and argon. In this paper experiments carried out in low atomic number $(\mathrm{Z}<26)$ plasmas, to study ionization potential depression (IPD) and line-shapes, are described along

Atomic Processes in Plasmas APiP 2016

AIP Conf. Proc. 1811, 050001-1-050001-9; doi: 10.1063/1.4975721

Published by AIP Publishing. 978-0-7354-1479-2/\$30.00 
with measurements of the L-shell spectra from germanium. At high density the electrostatic field in the plasma, due to the close proximity of ions, affects the binding energy of outer electrons, lowering it significantly compared to that in an isolated ion. In the last few years results from X-ray free electron laser experiments at the Linac Coherent Light Source (LCLS) [11, 12] have renewed interest in ionization potential depression along with results obtained over the same period from the Orion facility $[13,14]$. In the Orion experiments the ionization potential depression is diagnosed from the disappearance of aluminium line emission in He-like and H-like ions due to the upper levels becoming delocalized. These experiments are described briefly. Another aspect of dense plasma spectroscopy is the increasing effect of the plasma electrostatic field in the broadening and shifting of levels with increasing density via the Stark effect. Examples are given where the standard theory of line broadening combining a quasi-static microfield and electron impact broadening [15] must be augmented by impact broadening by ions in multi-species plasma and the inclusion of higher order than dipole terms in the plasma microfield. The K-shell line emission from chlorine and scandium high density plasmas is presented to illustrate this. Finally comparisons to the L-shell spectra of germanium are presented and compared with atomic physics models using both collisional radiative equilibrium (CRE) to calculate populations and state-of-the-art local thermodynamic equilibrium (LTE) opacity codes. The validity of comparisons of non-LTE data with LTE simulations are discussed.

\section{EXPERIMENTS ON DENSE PLASMA EFFECTS IN LOW Z MATERIALS}

The experiments described used one of the two Orion short pulse laser beams converted to second harmonic ( $2 \omega$ $0.53 \mu \mathrm{m})$ to heat solid density foils with buried dot samples. The effect of pre-pulse on the density scale-length seen by the main short pulse has a dramatic effect on the heating possible at a given laser energy as described in refs [14, 16]. Due to the requirement for a thin (2mm) type $1 \mathrm{KDP}$ conversion crystal to prevent back-conversion, the diameter of the beam that can be converted using a single crystal is limited. On Orion the full aperture of the short pulse beam is $60 \mathrm{~cm}$ in the fundamental but for second harmonic this aperture is reduced to $30 \mathrm{~cm}$ because this is the limit of the KDP crystal diameter. This aperture reduction, along with losses due to the conversion, limits the second harmonic energy to $100 \mathrm{~J}$ currently.

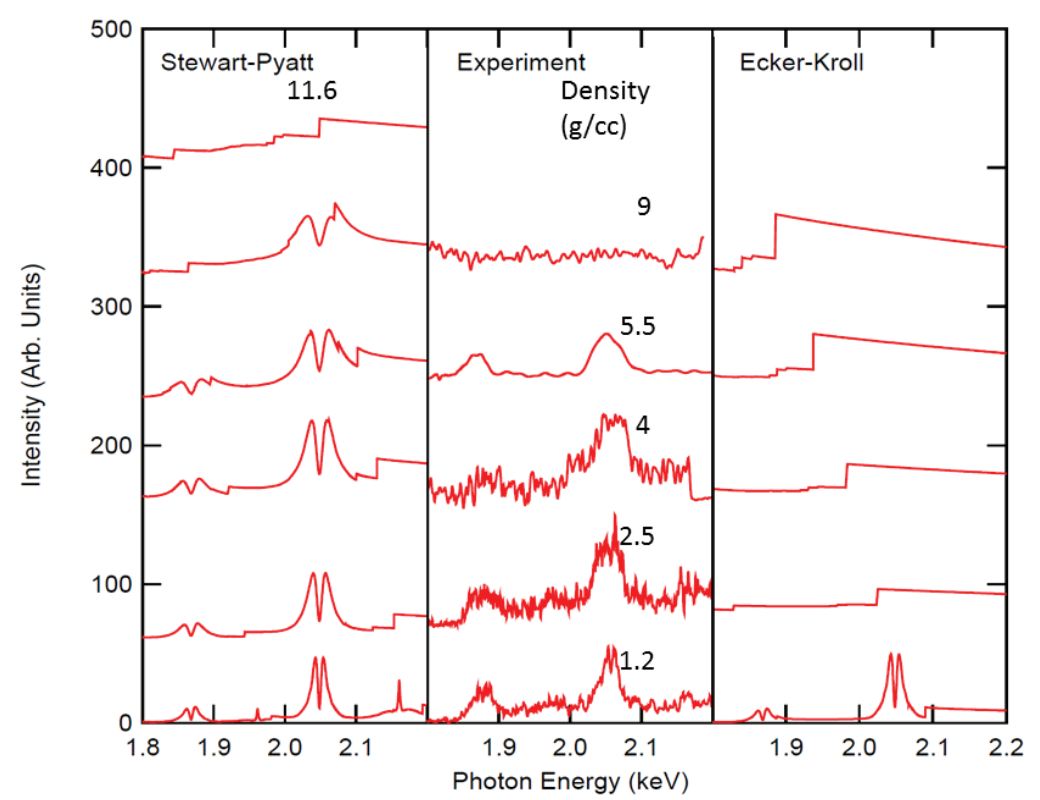

FIGURE 1. The central panel shows the results from Orion compared to an analytical model suggested by XFEL results on the right panel and widely used analytical modelling due to Stewart and Pyatt on the left panel.

Such is the effect of the improved pulse contrast that the reduction from 500J to $100 \mathrm{~J}$ in going from fundamental to second harmonic wavelengths is offset by the improved energy coupling to the solid target [16]. The arrangement of beams on Orion means that the target foil can be irradiated on one face with the short pulse beam converted to $2 \omega$, and on the opposite side with the long pulse beams shaped to have a $120 \mathrm{ps}$ rise. The long pulse beams drive a shock through the sample dot compressing it to between three and four times solid density. By firing 
the short pulse beam before the shock breaks out of the front face of the target the compressed sample can be rapidly heated and its emission spectrum recorded. By changing the material of the tamping foil from parylene $\mathrm{N}$ plastic (density $1.2 \mathrm{~g} / \mathrm{cc}$ ) to diamond and diamond-like carbon (density 3.5 and $3 \mathrm{~g} / \mathrm{cc}$ respectively) and taking measurements with and without long pulse compression a series of measurements at different densities were made of the K-shell emission spectrum of aluminium. Fig. 1 summarizes the experimental findings from the streaked aluminium spectra for the $\mathrm{n}=1-3$ line transitions in He-like aluminium (line at $1.86 \mathrm{keV}$ ) and the $\mathrm{H}$-like $\mathrm{Al}$ (line at $2.048 \mathrm{keV}$ ). At the highest density in the study $9 \mathrm{~g} / \mathrm{cc}$, three times solid density aluminium, the $\mathrm{n}=3$ level is no longer bound in He-like and H-like aluminium ions due to ionization potential depression and the $\mathrm{n}=1-3$ line emission disappears. The remaining line transitions from the $1 \mathrm{~s} 2 \mathrm{p}-1 \mathrm{~s}^{2}$ and $2 \mathrm{p}-1 \mathrm{~s}$ lines were used to infer the temperature and density. The atomic kinetics and spectral synthesis code FLYCHK [17] was used to compare an analytical model due to Ecker and Kröll [18] suggested by the Oxford/Berkeley group based on fitting their results [11] and the widely used model due to Stewart and Pyatt [19]. The Orion results clearly are poorly reproduced by the Ecker-Kroll model and though closer to Stewart and Pyatt differ from that model to a lesser extent. The Orion results are best reproduced by an ion sphere potential model in the CASSANDRA opacity code [20].

The plasma environment at high density has effects on the width and position of spectral lines which was exploited in the IPD experiments to infer the density. Though these densities were consistent with radiationhydrodynamics predictions using the NYM code [21] more experiments to confirm the line-shape theory would give further confidence in the densities inferred from fits to line-widths. Experiments to date have tended to be in low $\mathrm{Z}$ materials such as hydrogen, helium and carbon studied in rf-discharges in shock tubes, arcs and pinch discharges at electron densities below 1e19/cc or, at higher densities, in laser driven capsules where there are large gradients $[15,22]$. Investigations of the validity of densities inferred from line-shapes for high density strongly coupled plasma have been carried out on Orion using a layer of parylene C, a plastic with $50 \%$ by particle number of chlorine, buried in a foil of parylene $\mathrm{N}$ plastic, which is purely $\mathrm{CH}$. This is done to ensure no density change occurs on heating as the buried layer expands into the surrounding tamper material if there is a density mismatch. The data were compared to the FLYCHK model which is widely used in the plasma atomic physics community and another atomic kinetics spectral synthesis code ALICE due to Hill and Rose [23]. Data were recorded using a variety of spectrometers including an ultra-high resolution spectrometer called OHREX developed for Orion by Beiersdorfer et al. [24]. This recorded the chlorine $\mathrm{He}_{\beta}$ line and satellites at high resolution and was modelled using the ALICE code. The simulations with the ALICE code infer an electron density of $3 \mathrm{e} 23 / \mathrm{cc}$. These results will be described in detail elsewhere but here some aspects of the modelling of low $\mathrm{Z}$ spectra are presented. The ALICE code uses the quasi-static approximation; electron impact broadening and also a model of ion dynamic broadening based on the frequency fluctuation model (FFM) $[25,26]$ to calculate line-shapes. Including ion dynamics has the effect of slightly filling the central dip in the line profile of chlorine $\mathrm{He}_{\beta}$ due to the ion dynamics of the light ions from the plastic compared to a chlorine only plasma but leaves the gross line-shape essentially unchanged.
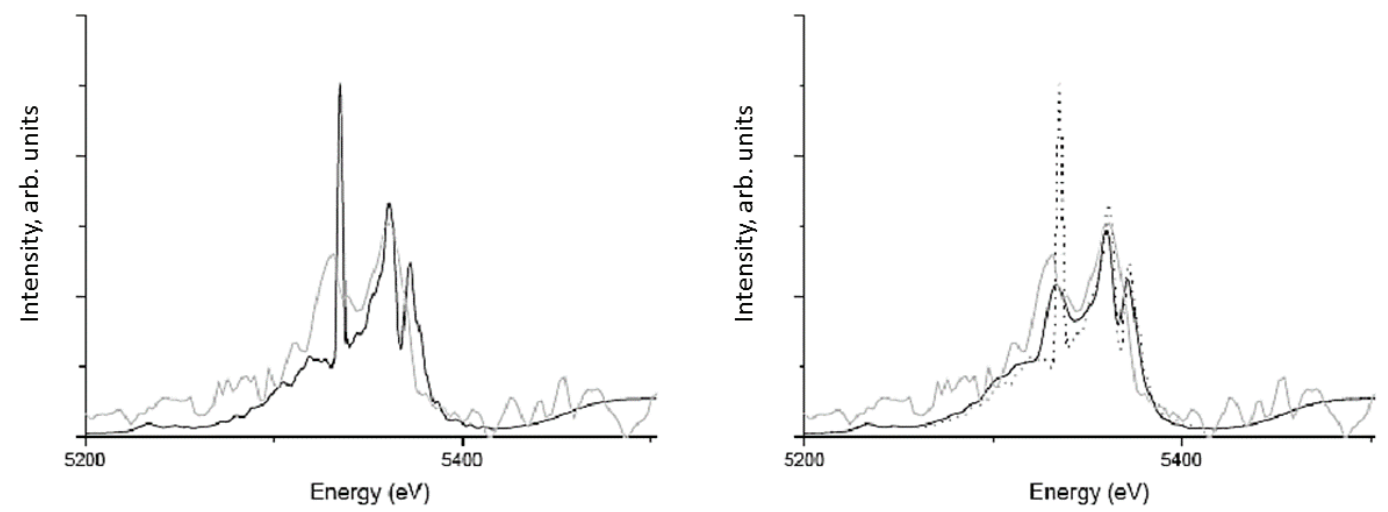

FIGURE 2. Scandium spectra from a buried microdot in plastic compared to ALICE simulations with dipole only terms in the microfield (left panel solid black line), and ALICE including higher order terms (solid black line in right panel). The agreement between simulation and experiment is significantly improved by inclusion of higher 
than dipole terms in the plasma microfield. The dipole only case is shown in the right hand graph as the dotted curve.

Further effects of line shape modelling are seen when comparing ALICE simulations with the spectra measured from other low $\mathrm{Z}$ experiments. Figure 2 shows the results of short pulse heated scandium in plastic measured with a PET crystal spectrometer recording onto a BAS-TR Fuji image plate. The initial fit to the $\mathrm{He}_{\gamma}$ line ( $\mathrm{n}=4-1$ transition) and the Lyp line shows a large disagreement with the He-like line when the microfield is calculated on the basis of dipole fields only. When quadrupole and higher order terms are included in the plasma microfield there is good agreement between experiment and simulation. This effect is also seen in the $\mathrm{He}_{\gamma}$ line in aluminium under similar conditions

\section{MEASUREMENTS OF EMISSIVITY IN HOT DENSE PLASMA}

The study of the plasma opacity through absorption and emission of radiation from hot, dense plasma presents a number of challenges. Conventional transmission measurements [27] require a back-lighter source significantly brighter than the sample self-emission. At temperatures $>200 \mathrm{eV}$ such a back-lighter has not been developed and so high temperature measurements of spectral structure are restricted to emission measurements at present. Emission measurements enable a wealth of information on the frequency dependence of the material opacity but except for low $\mathrm{Z}$ elements the sample plasmas, even at solid density or above, tend to be out of local thermodynamic equilibrium (LTE). In short pulse heated buried layer targets there are also temporal and radial gradients which have to be measured accurately. Nevertheless such experiments yield data which can validate both non-LTE and LTE models in temperature and density regimes to an accuracy not possible previously. Furthermore as nonLTE models have increased in accuracy and now approach the spectral fidelity seen in LTE opacity codes comparison to nonLTE experiments can inform both non-LTE and LTE modelling.

The use of microdot targets allows the measurement of plasma emission from a well-defined area. The mitigation of radial gradients is done by defocusing the laser spot to a diameter larger than the microdot diameter. The diameter of the microdot is chosen depending on the available laser energy, the signal level, diagnostic sensitivity and the change in the peak temperature with radius. In Orion experiments the radial gradient was measured as a function of time using a slit imager coupled to a picosecond resolution Axis-photonique x-ray streak camera to record the emission from $1.5-1.8 \mathrm{keV}$ with a peak around $1.7-1.8 \mathrm{keV}$ close the aluminium $\mathrm{Ly}_{\alpha}$ line. The imager used a $5 \mu \mathrm{m}$ slit and a $3^{\circ}$ mirror of $50 \mathrm{~nm}$ platinum on a quartz substrate. The tests were carried out on dots of 50,75 and $100 \mu \mathrm{m}$ diameter at best focus and with the $2 \omega$ beam defocused to a $50 \mu \mathrm{m}$ spot. The results showed the diameter of the emitting area did not change over time with a duration of emission from aluminium recorded at 2530ps. Measurements also showed the emitting area was significantly larger than the focal spot of the laser at best focus $(10 \mu \mathrm{m}$ laser spot resulted in $25-30 \mu \mathrm{m}$ area of X-ray emission measured) at a $50 \mu \mathrm{m}$ defocus the spot was similar to or slightly larger than the laser spot. The peak temperature variation as a function of radial position was measured using a spatially imaging x-ray crystal spectrometer with a resolution of $15 \mu \mathrm{m}$ recording time-integrated spectra onto BAS-TR Fuji image plate. These measurements were carried out using aluminium continuous layers and scandium continuous layers. The aluminium results showed a variation of less than $100 \mathrm{eV}$ across the spot but the results were repeated with scandium to check that variations in the peak temperature were not being missed due to the insensitivity of the aluminium to changes above $800 \mathrm{eV}$. The scandium results for the laser spot of $50 \mu \mathrm{m}$ showed a variation in peak temperature across the spot larger than recorded in aluminium. At various points in the spatially resolved spectrum lineouts were taken and fitted using the temporal history. The temporal data were calculated using the NYM radiation-hydrodynamics code treating the absorbed energy as a free parameter and using the ALICE and FLYCHK atomic physics models to generate synthetic spectra. These time histories were compared to measured time histories recorded with a $1 \mathrm{ps}$ resolution streak camera coupled to a crystal spectrometer. The EPOCH-CORVUS-THOR hybrid Particle-in-Cell and radiation-hydrodynamics model [28] was used to calculate the temporal and radial gradients from first principles using the measured energy onto target and the measured pulse length with good agreement with the temperatures and densities inferred from the NYM simulations, within the errors of the measured pulse-length and energy. Fig. 3 shows the schematic of the measurement and the variation in inferred peak temperature. The measurements show just under a $200 \mathrm{eV}$ gradient over the dot and the rapid roll-off at the edges of the spot. 

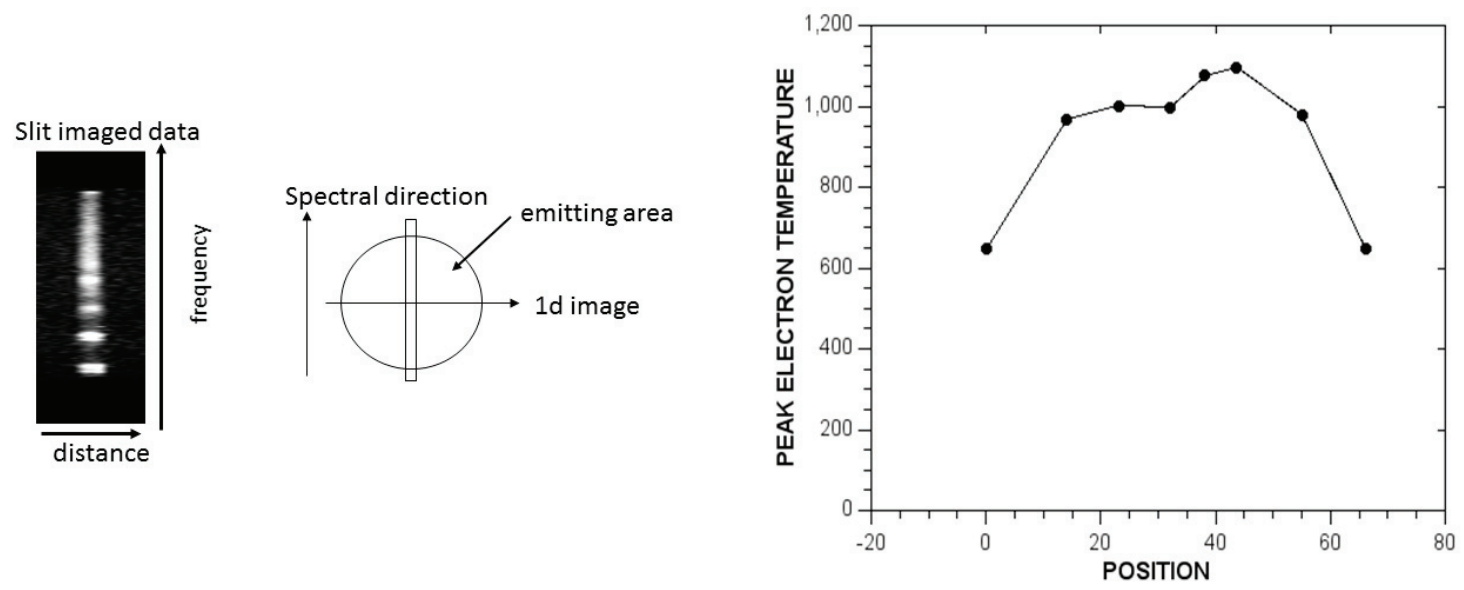

FIGURE 3. The variation in peak temperature across the emitting area of the defocused spot from a measurement of the short pulse laser defocused to a $50 \mu \mathrm{m}$ spot on a continuous buried layer of scandium shown in the graph on the right. The inferred peak temperature is given as a function of position across the spot in microns. The peak temperature varies across the spot by around $170 \mathrm{eV}$ then rolls off rapidly at the edge of the spot. An example image of a spatially resolved spectrum from aluminium is shown on the left with a schematic of the set-up showing the spectral and radial directions.

By ensuring that the microdot is smaller than the laser spot the rapid roll-off in temperature can be eliminated from the sample leaving a gradient of $150-200 \mathrm{eV}$ depending on the structure over the laser spot. This was done in the measurements described in the earlier sections of this paper and was applied to the measurements of emissivity to attempt to reduce gradients. The extent of this roll-off is under investigation with future measurements planned at higher resolution.

An example measurement of emissivity and comparison to model calculations of emissivity and opacity is given in the case of the L-shell spectrum of germanium. The samples were a mixture of germanium and a low $\mathrm{Z}$ element, titanium or aluminium which provide the spectral information to allow density and temperature to be inferred. The example given is from germanium compressed by a shock driven by a long pulse beam and heated with a high contrast short pulse beam at second harmonic as described above. The sample conditions were inferred as $4 \mathrm{~g} / \mathrm{cc}$ with a peak temperature of $950 \mathrm{eV}$. The measured spectrum is shown in fig. 4.

The experimental data can be compared to calculation using opacity codes assuming local thermodynamic equilibrium by evoking a temperature for the LTE calculation [29] which reproduces the ionization in the non-LTE experiment at the experimental density as shown in equations 1 and 2 below

$$
\begin{aligned}
& Z_{\text {NLTE }}^{*}\left(T_{e}\right)=Z_{L T E}^{*}\left(T_{z}\right) \quad T_{e}>T_{z} \\
& \kappa\left(T_{e}, Z_{N L T E}^{*}, \rho\right)=\kappa\left(T_{z}, Z_{L T E}^{*}, \rho\right)
\end{aligned}
$$

However, not only the mean ionization but the population distributions $p$ must be reproduced to give a meaningful comparison to LTE simulations using effective temperature $T_{\mathrm{z}}$ since :-

$$
\kappa \propto f_{i j} p_{i}\left(1-p_{j}\right)
$$

where $\kappa$ is the opacity, $f$ is the oscillator strength and $p$ is the level population. 


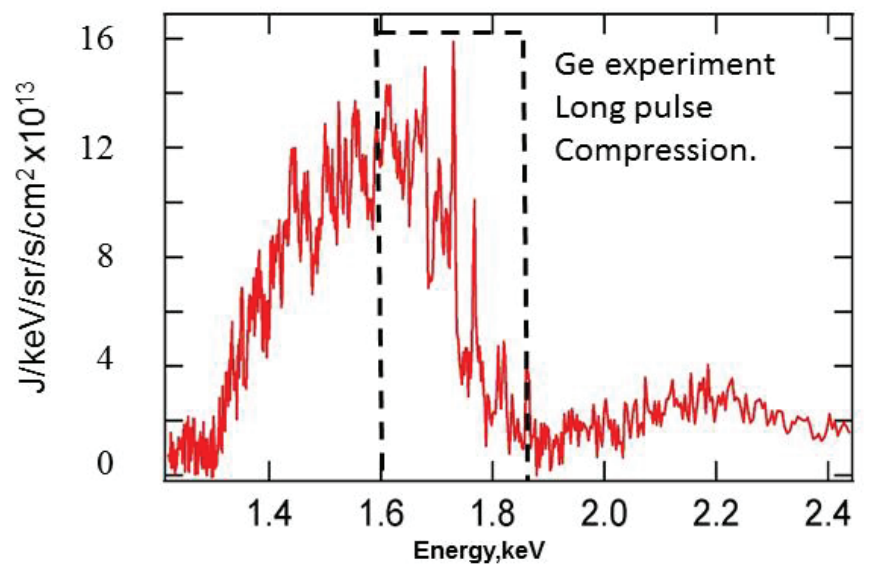

FIGURE 4. Compressed germanium emission spectrum. The section inside the dashed box is reproduced in fig.6 compared to simulations.

Fig. 5 shows the simulated populations using a steady state collisional radiative equilibrium (CRE) model called Aurora based on the average atom approximation with levels represented as superconfigurations similar to the nonLTE opacity code SCROLL [30].
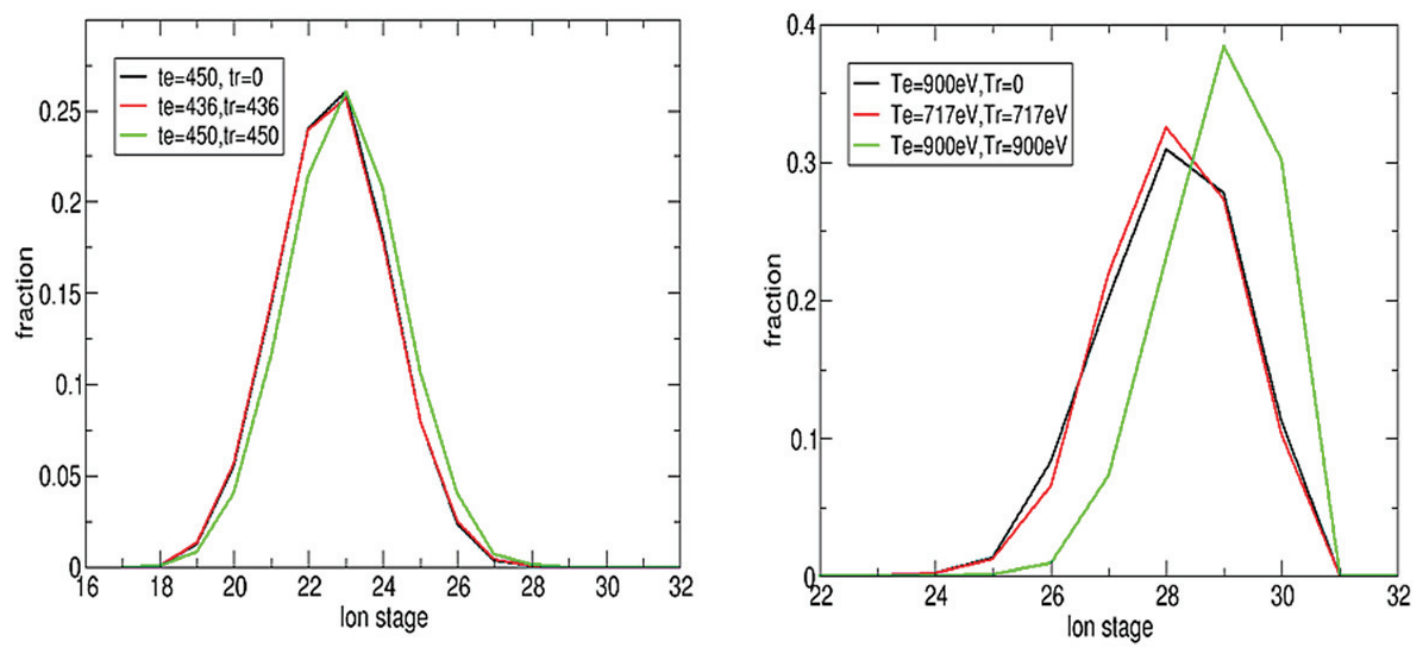

FIGURE 5. Population distribution for effective temperatures compared to LTE and non-LTE cases. Case 1 is the left panel; case 2 is the right panel (see text).

Two examples are given for germanium which include a worse case of the non-LTE plasma with $\operatorname{Tr}=0$ i.e. no internal radiation field. In practice this is never the case since the opacity sample, though not optically thick, does have a finite thickness and a non-zero radiation field. Case 1 shows ionization distributions for a germanium plasma at $450 \mathrm{eV}, 4 \mathrm{~g} / \mathrm{cc}$ for the LTE case; an effective temperature of $436 \mathrm{eV}$ in the LTE and shows how well this reproduces the ionization and populations of the non-LTE case at $450 \mathrm{eV}$. This shows a temperature difference between the electron temperature and effective temperature of $14 \mathrm{eV}$. Case 2 shows the effect at higher electron temperature of $900 \mathrm{eV}$. In this case the effective temperature of $717 \mathrm{eV}$ reproduces the non-LTE ionization however, there is a slight difference in population distribution at the effective temperature compared to the non-LTE case with zero radiation field. This difference is not enough to affect the spectrum significantly and when compared to experiment is within temperature experimental error. This also represents a worse case since no radiation field is included in the CRE model. 
Fig 6 shows the comparisons of the germanium data and two LTE models calculated at effective temperatures covering the gradients in the experiments, up to a peak of $800 \mathrm{eV}-150 \mathrm{eV}$ lower than the peak electron temperature inferred from the titanium line ratios. Part of the experimental spectrum showing the Li-like germanium $2 p-3 d$ features is expanded for illustration. The codes against which the data are compared are a detailed configuration accounting model, CASSANDRA [20] and a detailed line accounting model called DAVROS [31]. The comparisons clearly indicate the smoothing of spectral structure by the detailed configuration accounting model but also show a systematic shift in the line positions in CASSANDRA which has been identified as a discrepancy in the treatment of the exchange potential and has now been rectified [32] in the CASSANDRA model.

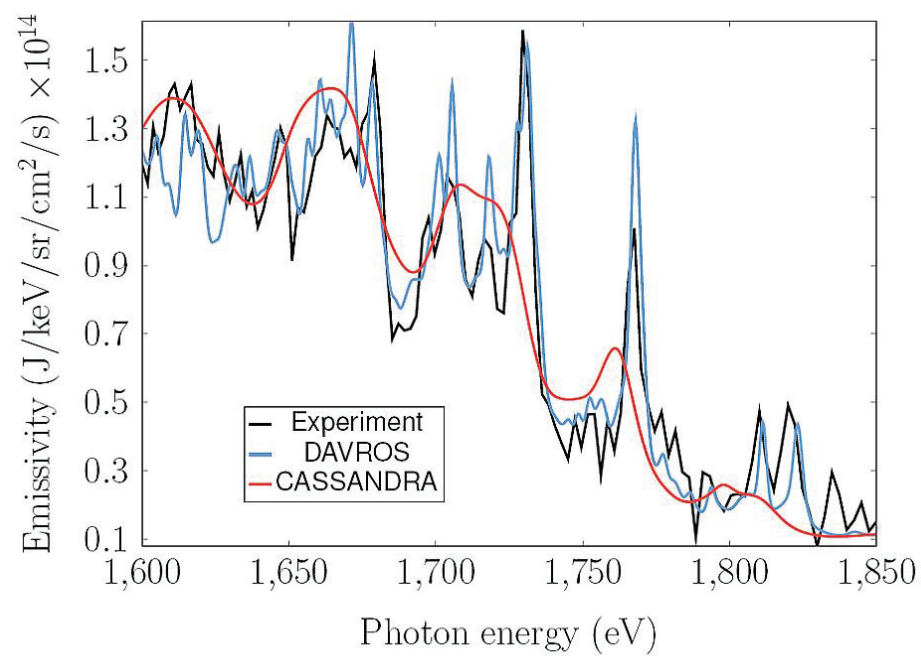

FIGURE 6. Comparisons of germanium experimental data with LTE simulations by the CASSANDRA and DAVROS models.

\section{SUMMARY/CONCLUSION}

Buried microdot targets heated by a short pulse high contrast laser pulse have proved to be a useful testbed for hot, dense plasma and, when combined with long pulse compression and higher density tampers such as diamond, have allowed an extension of measurements to much higher densities than previously possible. By studying embedded dots, at similar density to the tamper material, hydrodynamic expansion of the buried layer into the surrounding material can be minimized removing changes in density until comparatively late times after the X-ray emission pulse from the sample has been measured. Dense plasma effects such as ionization potential depression have been studied and experiments on chlorinated plastic tamped in plastic, and scandium in plastic, have shown the effectiveness of this platform in studying aspects of line-broadening. By characterizing the temporal and radial gradients complex L-shell spectral measurements can be made with an accuracy which allows comparison of experimental data to simulation predictions. These comparisons can be made to both non-LTE models of opacity and to models assuming local thermodynamic equilibrium by using an effective temperature approach, within certain limits, where the perturbation of bound electron population distributions from non-LTE experimental conditions and the LTE model at an effective temperature are small. These data are at conditions found in stellar interiors and are relevant to the calculation of the material properties of elements and mixtures found in the solar radiative zone. Fig. 7 shows the radial variation of temperature and density in the solar interior and also an example showing the contributions to the solar opacity at example conditions in the radiative zone. The Orion experiments are capable of temperatures exceeding those in the radiative zone and even the solar core but the challenge is to access the very high densities simultaneously. The planar compression experiments have shown that dense, hot Lshell spectra from germanium can be studied. Germanium is analogous to the L-shell of iron at solar conditions and the chlorinated plastic experiments are in some respects, analogous to the argon in the multi-species mixture found in the solar interior. Further experiments are planned which use high density tampers and cylindrically convergent geometries to extend these measurements to higher density. 

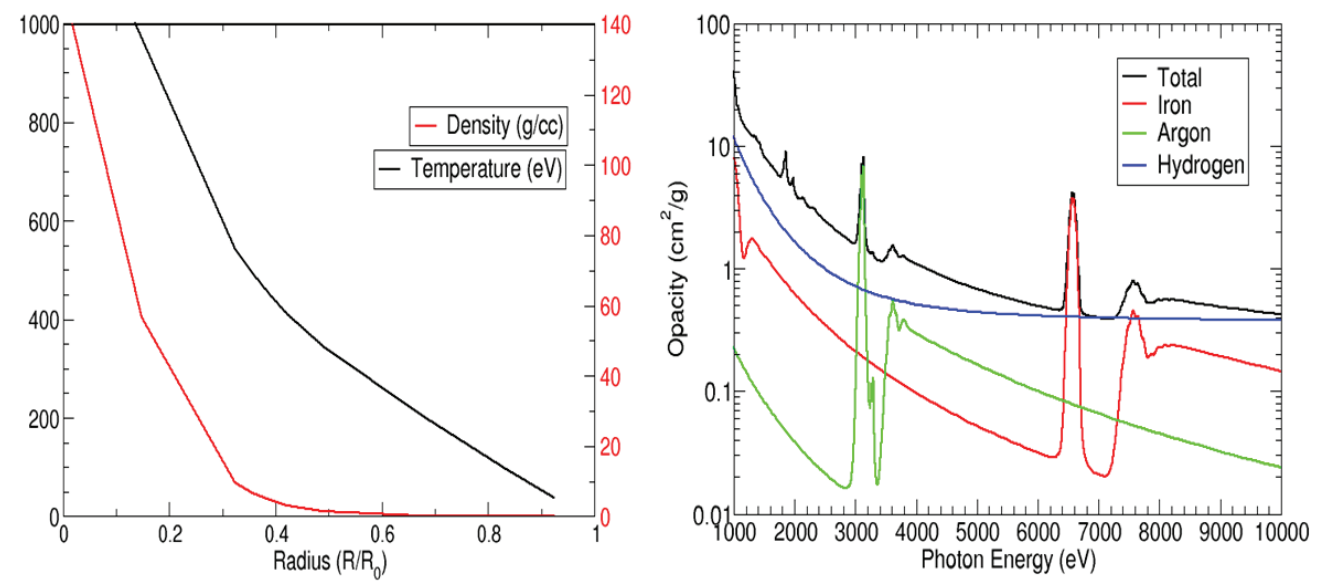

FIGURE 7: Conditions in the solar interior as a function of radius shown in the left graph and, on the right, the contributions to the opacity of different elements at a representative condition in the radiative zone of $500 \mathrm{eV}$ and $6.5 \mathrm{~g} / \mathrm{cc}$.

\section{ACKNOWLEDGMENTS}

The authors would like to acknowledge the help and dedication of the staff of the Orion laser and AWE target fabrication.

\section{REFERENCES}

1. D. Salzmann, Atomic Physics in Hot Plasmas, (Oxford University Press, New York, 1998)

2. R. P. Drake, High-Energy-Density Physics (Springer, Berlin Heidelberg, 2006)

3. S. J. Rose, Contemp. Phys. 45, 109 (2004)

4. J. D. Lindl, Inertial Confinement Fusion (AIP Press, Springer, Berlin Heidelberg New York, 1998)

5. K Nazir et al., Appl. Phys. Lett. 69, 3686 (1996)

6. B. K. F. Young et al., Phys. Rev. E, 58, 4929 (1998)

7. A. Saemann et al., Phys. Rev. Lett. 82, 4843 (1999)

8. K Eidmann et al., J. Quant. Spectrosc. Radiat. Transfer 81, 133 (2003)

9. R. G. Evans et al., Appl. Phys. Lett. 86, 191505 (2005)

10. V. Dervieux et al., High Energy Density Phys. 16, 12 (2015)

11. O. Ciricosta et al., Phys. Rev. Lett. 109, 065002 (2012)

12. O. Ciricosta et al., Nat. Commun. 7, 11713 (2016)

13. D. J Hoarty et al., Phys. Rev. Lett. 110, 265003 (2013)

14. D. J. Hoarty et al., High Energy Density Phys, 9, 661 (2013)

15. H. R. Griem, Principles of Plasma Spectroscopy, (Cambridge University Press, Cambridge 1997)

16. C. R. D. Brown et al., Phys. Rev. Lett. 106, 185003 (2011)

17. H. K. Chung et al., High Energy Density Phys, 1, 3 (2005)

18. G. Ecker and W. Kröll, Phys. Fluids 6, 62 (1963)

19. J. C. Stewart and K. D. Pyatt Jr, Astrophys. J. 144, 1203 (1966)

20. B. J. B. Crowley and J. W. O. Harris, J. Quant. Spectrosc. Radiat. Transfer, 71, 257 (2000)

21. P. D. Roberts et al., J. Phys. D 131957 (1980)

22. H. J. Kunze, Introduction to Plasma Spectroscopy, (Springer, Heidelberg Dordrecht London New York 2009)

23. E. G. Hill and S. J. Rose, High Energy Density Phys. 8, 307 (2012)

24. P. Beiersdorfer et al., Rev. Sci. Instrum. 87, 063501 (2016)

25. B. Talin, A. Calisti, L. Godbert, R. Stamm and R. W. Lee, Phys. Rev. A 51, 1918 (1995)

26. C. A. Iglesias, High Energy Density Phys. 9, 209 (2013)

27. T. S. Perry et al., Phys. Rev. E 54, 5617 (1996)

28. N. Sircombe et al., J. Phys. Conf. Series 717, 012081 (2016) 
29. M. Busquet, Phys. Fluids B 54191 (1993)

30. A. Bar-Shalom et al., J. Quant. Spectrosc. Radiat. Transfer, 58, 427(1997)

31. L. M. Upcraft, M. Jeffery and J. W. O. Harris, High Energy Density Phys. 14, 59 (2015)

32. M. Jeffery, J. W. O. Harris and D. J. Hoarty, High Energy Density Phys. 20, 1 (2016) 